\title{
Dispositional and situational attribution of COVID-19 risk: A content analysis of
} response typology

\author{
Alice Dunning ${ }^{1,2}$ \\ Elizabeth Travis ${ }^{1}$ \\ Madeleine Pownall ${ }^{*}$
}

1. School of Psychology, University of Leeds, Leeds, UK, LS2 9JZ

2. Bradford Institute for Health Research, Bradford, BD9 6RJ

*Correspondence: Madeleine Pownall, M.V.Pownall@leeds.ac.uk

Conflict of Interest. The authors declare that the research was conducted in the absence of any commercial or financial relationships that could be construed as a potential conflict of interest.

Author Contributions. AD and MP applied for ethical approval, collected study data, and conducted the first coding of the data. AD coded the data for response typologies and MP coded a random $10 \%$ of responses. ET coded the full dataset. All authors contributed equally to the writing, editing, and preparation of the manuscript.

Funding. No funding was received for this work.

Data Availability Statements. The datasets generated for this study can be found in the Open Science Framework Project Page:

https://osf.io/vseu5/?view_only=e8318ba4e1b24ccfa254f3a8912345ae 


\begin{abstract}
:
In the current COVID-19 pandemic, there have been official health recommendations of social distancing, thorough handwashing, and self-isolation. However, compliance with these recommendations has been mixed. We suggest that non-compliance may be justified by one's (mis)perception of their own COVID-19 risk. In this paper, we explore the dispositional and situational attribution of self-reported COVID-19 risk, as per Heider's Attribution Theory. We conducted a content and framework analysis of responses to an online survey, in which participants $(N=114)$ were asked to rate their likelihood of contracting the COVID-19 virus, before providing textual responses to explain their rating. Overall, we observed that generally, participants who more dispositional attributions (i.e., attributing their risk to factors such as age and own personal hygiene) rated their COVID risk to be significantly lower, whereas participants who made more situational attributions (i.e., attributing COVID19 risk to government decision-making and other people's cleanliness) rated their risk significantly higher. Following analysis of the textual data, a finalised framework of five response typologies including intrinsic, behavioural, balanced, contextual, and disengaged responses was systematically applied to the data. These results will be discussed in the context of risk perception, whilst providing future recommendations for research into COVID-19 behaviours.
\end{abstract}

Keywords: COVID-19, CORONAVIRUS, ATTRIBUTION THEORY, RISK PERCEPTION, HEALTH BEHAVIOUR 


\section{Dispositional and situational attribution of COVID-19 risk: A content analysis of response typology}

COVID-19 continues to affect the daily lives of people around the world at alarmingly increasing rates (NHS, 2020). The UK government and official health authorities have advised that behaviours such as handwashing, social distancing, and self-isolation can mitigate the effects of the spread of COVID-19 (Ferguson et al, 2020). However, compliance with these recommendations has been generally inconsistent (Oosterhoff \& Palmer, 2020; Sobol et al., 2020) with many people neglecting the severity of the global pandemic and even, in extreme cases, denying its existence (Stanley, Seli, Barr, \& Peters, 2020). Compliance to COVID-19 behavioural guidelines has been related to self-interest and health concerns (Murphy et al., 2020), suspensibility to misinformation (Roozenbeek et al., 2020), and perceived risk (Wong et al., 2020). As Kuper-Smith et al (2020) recently reported, misperceptions about the severity of COVID-19 may be due to their observed 'optimism bias', in which the general population underestimates their own individual risk of contracting the COVID-19 in relation to the rest of the population. Such biases in risk perceptions have been noted in other historical pandemics, such as influenza (Xu \& Peng, 2015).

To add to this, in a recent summary of the social psychological implications of COVID-19, Van Bavel et al., (2020) note that the proliferation of conspiracy theories, fake news, and a general mistrust of health official advice means that it is now increasingly difficult for the general public to fully and meaningfully engage with the COVID-19 health risk. In the context of these widespread biases and misperceptions, it is important to understand how people assess their own risk of contracting COVID-19. Preliminary work has begun to investigate this. For example, in a recent preprint, Gerhold (2020) noted that, in a German sample, demographic factors such as gender and age influence self-reported 
perceptions of individual COVID-19 risk (see also Bruine de Bruin, 2021). These risk perceptions may contribute significantly to the effective combat of COVID-19 in that a more accurate understanding and attribution of risk may improve engagement with official health advice (e.g., Tenforde et al., 2021).

Here, we propose that the broad principles of Attribution Theory (Heider, 1958), may enable us to explore attributions of risk in the context of the current COVID-19 pandemic. Attribution Theory dictates that we are motivated to attribute meaningful causes to action and behaviour (Kelley, 1967, 1971). The theory suggests that when faced with the task of appraising a situation, we broadly make either dispositional attributions, which reside firmly within the individual, or situational attributions, which refer to external factors outside of the individual (Valins \& Nisbett, 1971). Our assessments about the aetiology of behaviours and actions, including health outcomes (Marteau, 1995), are also informed by factors such as attribution stability and controllability (Kelley, 1967; Weiner, 1985).

Moreover, these attributions of risk can generally reside inside or outside of one's 'locus of control' (Wallston, 1992). Health locus of control, in this sense, is the extent to which one's behaviour is perceived as being related to eventual health outcomes (Rotter, 1954). Individuals with an internal locus of control perceive a stronger relationship between their own behaviour and subsequent health outcomes, and thus have a strong sense of healthrelated autonomy (Rowe et al., 2005). Those with external locus of control attribute health outcomes to external factors, such as health profession competence, medication, and situational factors.

The notion of health locus of control largely echoes Janoff-Bulman's (1979) assertion that there are two forms of 'self-blame' in health contexts: 'characterological', which occurs when health outcomes are attributed to stable, internal characteristics, and 'behavioural', which views one's own modifiable behaviour as negative. Endorsement of internal locus of 
control has previously been associated with positive health behaviour change (Norman, Bennett, Smith \& Murphy, 1998; Seeman \& Seeman, 1983), in that they are associated with higher expectations for health-related coping (Alexrad, 1982; Weiner, Nierenberg \& Goldstein, 1976) and higher levels of compliance with health recommendations (Bar-on, 1983; Maddux, Brawley, \& Boykin, 1995; O’Hea et al., 2005) This all fits neatly into Heider's attribution theory (1958); internal locus of control may be synonymous with characterological forms of self-blame and thus dispositional attributions, whereas external locus of control, may be generally more aligned with an behavioural or situational attribution of health risk.

The extent to which a person demonstrates an internal or external health locus of control also informs their attribution style and thus their approach to relevant health outcomes. For example, an 'optimistic' explanatory style, whereby people appraise prior negative events in a way that focuses on the external, malleable, transient aspects of the event, rather than fixed, global causes (Gillham et al., 2002; Peterson \& Villanova, 1988) is associated with better health outcomes (Peterson, Seligman, \& Vallaint, 1988; Hirsch et al., 2009). In the context of COVID-19, therefore, it is important to establish how people approach the task of judging their likelihood of contracting the virus, through a lens of explanatory styles and loci of control.

Beliefs related to perceived health control, self-blame, and thus, risk attribution all may be wholly important factors to consider in the context of improving uptake of COVID19 health behaviours. With this in mind, people's explanatory style, or the typology of their risk perception, is of importance in the context of COVID-19 health. This can be best understood through a lens of Schulman, Catellon and Seligman's (1989) explanatory style methodology. The authors note that decisions about the likelihood of events may be explained by either an optimistic or pessimistic explanatory style (e.g. Abramson, Seligman, 
\& Teasdale, 1978). To demonstrate this, Schulman and colleagues provided 169 participants with an Attributional Style Questionnaire (ASQ) and conducted a content analysis of verbatim explanations in response to 12 hypothetical scenarios. This provides strong theoretical rationale for the present study, which utilises a similar methodology to investigate the relationship between explanatory style, or response typology, and perceived risk of COVID-19.

Our methodology in this cross-sectional study is informed by the work of Schulman, Castellon, and Seligman (1989), who provide guidelines for assessing explanatory style through the use of verbatim responses from participants. Although there has been much research in recent months which ask direct, explicit questions about COVID-related stress, risk, and coping (see Rajkumar, 2020; Brown et al.,2020; McBride et al., 2020, for recent reviews), this study utilises a more indirect, qualitative approach. Content analysis of textual data can be a useful pragmatic approach that allows the researcher to synthesise complex information on a well-defined topic (Kuchkartz, 2019). As per Shulman's (1989) guidelines, our approach here is deductive, in that we have a specified framework, namely Heider's Attribution theory and health locus of control, that we wish the appraise the data with.

In this paper, we explore the attribution of risk and associated health locus of control to individual risk perceptions of contracting COVID-19, through a lens of Heider's Attribution Theory (1958). This responds to O'Connor et al's (2020) call to action for psychological science's response to the COVID-19 pandemic. Our core research question aimed to investigate how people assess their own risk of contracting the COVID-19 virus, testing whether high or low risk is associated with more internal or external attributions. Specifically, we aimed to test, using content and framework analysis, how the attribution of perceived risk fits within broader typologies of risk responses, that may enable health officials to provide more targeted COVID-19 advice. 


\section{Method}

\section{Participants and ethics}

An opportunistic sample was recruited from the University of [anonymised for peerreview] undergraduate participant pool (47.37\%) or through Prolific Academic (52.63\%), which is an online survey platform whereby participants complete paid studies. Participants who were recruited on Prolific were paid the equivalent of $£ 7.00$ per hour for their time. Participants recruited via the participant pool were granted credit in exchange for their participation. Data for this study comprised of a larger study which broadly assessed COVID19 risk perception in the context of an experimental intervention that aimed to improve health behaviour adherence. All of the responses to items reported here were completed prior to the implementation of the online intervention in the wider study. This study was conducted online via the survey host Qualtrics, due to both recommendations to self-isolate at the time of data recruitment. Data collection was conducted from the 12th to 19th March 2020 with UK-based participants.

Of the 144 participants surveyed in the original full dataset, $121(84.03 \%)$ provided textual responses. Of these, six participants gave textual responses but did not complete the risk perception item, so their data were excluded from further analyses. 1 participant was removed for reporting that they had contracted COVID-19. This resulted in a final total of 114 participants.

Participants were mostly white (83.5\%) and female (76.5\%) with a mean age of 26.2 $(S D=10.74)$. All participants were UK citizens and the study was completed in English. Ethical approval was granted by the School of Psychology Ethics Committee on 11th March 2020 (Ref: PSYC-4).

\section{Procedure}


After providing informed consent, in which participants were told the study is interested in 'how health messages affect behaviour and stress', participants completed a demographics questionnaire, which included age, gender, and ethnicity. Within this larger dataset, participants were then asked questions about measures such as behaviour intention, attitudes, and self-efficacy, which are not reported or analysed here.

Participants were asked to indicate how likely they believed they were to contract the COVID-19 virus, on a 0 (not at all likely) to 100 (very likely) scale. To explore these responses further, participants were asked 'Can you explain why?' and were provided with an essay response box. They were then debriefed, provided with official COVID-19 resources, and thanked for their participation.

\section{Data analysis}

Deductive content analysis was conducted at first to find commonalities across responses (Krippendorff, 2004); and was guided by a conceptual framework (attribution theory). The initial coding was conducted by both the researchers (AD, MP) and consisted of two core codes: situational attribution and dispositional attribution. The definition of these codes was discussed between the two authors prior to coding commencement. A coding grid was created by one researcher (AD), with the responses in rows, and situational attribution, dispositional attribution, and other comments/notes, in which each column received a yes or no response from the researchers.

Driven by attribution theory, health locus of control, and health-related explanatory styles, each response was allocated a yes for situational attribution, where participants described external factors, including, for example: when participants attribute their risk to environmental characteristics (such as work or school), the actions of others, or contact with other people. Participants were allocated a yes for dispositional attribution if they described internal factors, including: their age, health choices, and their own compliance with 
preventative behavioural recommendations (e.g., handwashing, wearing a mask). If participants specifically mentioned abiding by the behaviours of hand washing, mask wearing or social distancing then they would be provided a yes under the relevant category. Any other health related behaviour, including, for example, broad reports of 'following government guidelines' were noted within the 'other behaviours' column. Under 'other comments' researchers provided further comments that were not captured within the previous categories.

We first coded the whole dataset as pertaining to either situational attribution, dispositional attribution, or both. Attribution types were not mutually exclusive, that is, participants could include both attributions per response. Two coders independently coded all responses using these two columns, and agreement between coders was initially $88.6 \%$ for responses coded as containing dispositional attributes and $91.23 \%$ for situational attributes. All authors then discussed and resolved any inconsistencies, resulting in a final agreement of $100 \%$ for both columns on the coding grid.

Following this, framework analysis was conducted. Once data familiarisation had been achieved, reflections and initial impressions of the similarities and differences between the responses were noted. Building upon the codes generated within the deductive content analysis, and these reflections an initial classification system was created, this initial system was refined during several iterations by reading and re reading the responses to ensure the final framework encapsulated all responses. Discussion between both researchers (AD) and (MP) during these iterations occurred to ensure agreement on the final framework and typology descriptions. This final framework of five response typologies was systematically used to categorise the data. A random ten percent of the responses were independently coded using this framework by another researcher (MP), as there was 100\% agreement on these cases. 
Finally, at this stage, a third, independent researcher (ET) then analysed the entire dataset for the final five typologies established, to determine the reliability of the coding procedure and robustness of the codes. The third researcher was independent from the initial research team and had no prior knowledge of the study or the research question, prior to this exercise. This followed Fereday and Muir-Cochrane's (2006, p. 90) recommendations of 'corroborating and legitimating coded themes' in deductive coding, in order to demonstrate rigour of the coding process. The original team first met to describe and discuss the five typologies, before the third researcher independently ascribed a typology to each of the 114 responses, blind to the original codes of the research team. Following this third round of coding, agreement of codes on all responses was 91.23\% (104 matches out of 114 responses). Cohen's kappa was 0.83 . All authors then met to discuss the 10 cases which were not matches. Of the non-matches, 3 cases were coded as a 'balanced' response by the third coder, and a 'contextual' response by the original coder.

Following analysis of the textual data, we then conducted a between-subjects ANOVA with response typology as the independent variable and overall risk of contracting COVID as the dependent variable. We also conducted two separate t-test to investigate the effects of attribution style of risk perception. The quantitative data analysis was conducted using statistical software JASP and aimed to provide robust evidence to the inferences made within the qualitative data. All data for this study can be openly accessed here: https://osf.io/vseu5/?view_only=e8318ba4e1b24ccfa254f3a8912345ae.

\section{Results}

\section{Textual results}

Participants wrote an average of 39 words in their responses $(S D=33$, Min $=4$ words). No participants were excluded for not writing enough, all participants provided 
responses that could be coded and analysed. There were five response typologies identified through framework analysis: Behavioural (12.28\%, 14 responses), Intrinsic (14.04\%, 16 responses) Balanced (25.44\%, 29 responses); Contextual (20.18\%, 23 responses) and Disengaged (27.19\%, 31 responses). All 114 responses fit within the framework within one response typology. The five response typologies are discussed in detail below.

\section{Behavioural response}

Here, participants, particularly mainly attributed their risk to dispositional factors $(85.71 \%)$, rather than situational (14.29\%). Within this response, participants viewed the risk of contracting COVID-19 as being nearly completely negated through their behavioural responses, thus reported a low perceived risk. As these behaviours were reported as being situated firmly within each participant's control, the agency of the respondent was clear. This response yielded the highest proportion in behavioural responses from each of the five typologies. For example, participants frequently reported using social distancing in response to the threat:

"I do not believe I will come into contact with anyone who has contracted it.

Furthermore I have been keeping up to date with all guidance to ensure I follow all the best steps." (Risk perception rating: 3\%)

"I'm currently remaining indoors at home to prevent family members at home from contracting it. Therefore, I feel that I'm unlikely to come into contact with it." (Risk perception rating: $35 \%$ )

\section{Intrinsic response}

The next typology of response was coded as an Intrinsic response, whereby participants attribute their COVID-19 risk to their own intrinsic characteristics. Within these responses, participants overwhelmingly attributed their COVID-19 risk to dispositional factors $(87.5 \%)$, 
rather than to situational factors $(37.5 \%)$. Here, responders mainly reported low or no risk of contracting COVID-19 and cited their inherent traits or characteristics as a means for justifying this risk. These included: overall health, their immune system and fitness behaviours. For example, most of the participants (62.5\%) explicitly mentioned their age as a perceived contributor to overall low risk. Participants who rated their risk to be low using an intrinsic typology of response typically stressed the ways in which they differ from high-risk groups:

"I believe that my chances of getting coronavirus are very low because I am healthy, have a strong immune system and I don't have any current underlying health issues that may attract the virus." (Risk perception rating: 8\%)

Intrinsic attribution responses were also made for those in the high-risk group, with some participants in this group also attributing their risk perception to underlying health conditions, as per this participant:

"I have a weak immune system which I think puts me at greater risk of contracting it, and definitely puts me at greater risk of not recovering from it". (Risk perception rating: $90 \%)$

\section{Balanced response}

Balanced responders also showed a high adherence to behavioural guidelines; this was the second highest of all the observed typologies. For example, some participants reported using hand washing in response to the COVID-19 risk, which contributed to their overall sense of individual risk. However, unlike the Behavioural response, participants did not view this as negating the risk, recognising the severity of the high contagion rates. Therefore, these participants showed a balance in dispositional $(77.42 \%)$ and situational (61.29\%) attributions: 
"Although I wash my hands frequently, it is spreading very fast so chances are quite high however not necessarily vicious when contracted" (Risk perception rating: 54\%)

\section{Contextual response}

The vast majority respondents in this response typology attributed their COVID-19 risk to situational attribution (87\%), rather than dispositional (47.87\%). Within this response typology, participants perceived themselves at a high risk and attributed this to external sources citing contextual justifications. The most common of these was the perception that other people demonstrate a lack of hygiene, and therefor contact with others was perceived to increase individual risk of contracting the virus:

"I'm a university student, and my university has already had one positive case. With people not covering the mouths when coughing, attending classes while ill and not washing hands or using hand sanitizer (all of which I have seen this week alone), it is a near guarantee I come into contact with it." (Risk perception rating: 100\%) More wider, societal factors, such as the contribution of media and the government's handling of the COVID-19 pandemic were also frequently cited within the contextual typology response:

"I believe my chances of contracting COVID-19 are extremely likely. This is because of how contagious it appears to be and the way the government is handling the situation. I think regardless of how much good hygiene I practice myself, I cannot particularly rely on other people to do the same." (Risk perception rating: 90\%)

\section{Disengaged response}

The majority of all participants across typologies and attribution type provided a disengaged response (27.19\%). These respondents were mainly categorised as showing situational attribution (86.21\%), compared to dispositional attribution (44.83\%). Disengaged 
respondents perceived their chances of contracting the COVID-19 to be inevitable and thus positioned themselves with little to no agency. Due to their perception that contracting COVID-19 is inevitable, participants reported a disengagement with behavioural responses which would reduce this risk, for example, this response typology gerally had the lowest proportion of participants reporting adherence to social distancing. These responses often cited government statistics about likelihood of contracting the virus and were consistently associated with higher risk perceptions:

"I think it's inevitable. It's just a question of when now. I believe that whilst the current measures will slow the spread, they aren't gonna stop it completely." (Risk perception rating: $100 \%$ )

\section{Inferential results}

\section{Response typology}

To test whether participants who had responses that were coded as either behavioural, intrinsic, balanced, contextual, or disengaged differed in their perception of their overall risk of contracting COVID-19, we ran an independent-groups analysis of variance (ANOVA), with typology as the independent variable and risk perception as the dependent variable. This revealed a significant main effect of typology on risk perception $F(4,109)=21.04 p<$ 0.0001, $\eta^{2}=0.44$. Post-hoc tests with Bonferroni corrections showed that there was no significant difference in mean risk perception between behavioural responses $(M=22.14, S D$ $=23.64)$ and intrinsic responses $(M=27.56, S D=23.44), \mathrm{p}=1.00$. There was also no significant difference between balanced $(M=55.5, S D=19.4)$ and contextual responses $(M=$ $68.21, S D=23.45)$, or contextual and disengaged $(M=73.5, S D=22.58)$. There were, however, significant differences between all other comparisons of typology groups $(p<$ 0.001; Table 1). Overall, contextual and disengaged responses were associated with higher 
risk perception, behavioural and intrinsic responses were associated with lower risk perception, and balanced responses were in between the two. Figure 1 shows the perception of COVID risk by response typology group. There was not a significant effect of gender on risk perception, $(t(111)=0.075, p=0.46)$, nor was there a significant correlation between participants' age and risk perception $(r=0.1, p=0.30)$.

\section{[Table 1 here]}

\section{[Figure 1 here, please]}

\section{Attribution type}

We then conducted two independent t-tests to test whether making dispositional or situational attributions was associated with COVID-19 risk perception. We conducted separate tests for each dispositional and situational attributions, given that one response could contain both kind of attributions. This analysis showed that participants whose responses were coded as featuring dispositional attributions had significantly lower risk perception scores $(M=45.23, S D=26.98)$ than those who $\operatorname{did} \operatorname{not}(M=72.5, S D=24.12) \cdot t(112)=$ 5.34, $p<0.001$, Cohen's $\mathrm{d}=1.05$. Likewise, participants who were coded as making situational attributions had significantly higher $(M=68.21, S D=22.62)$ risk perception compared with those who did not make situational attributions $(M=30.15, S D=21.74)$ $\mathrm{t}(111)=8.72, p<0.001$, Cohen's $\mathrm{d}=1.71$.

\section{Discussion}

This study aimed to assess how people attribute individual risk in the face of a global health pandemic, the COVID-19 pandemic. We coded the responses of 114 participants for dispositional (e.g., age, personal hygiene, pre-existing health conditions) and situational 
attributions (e.g., government handling of the virus, other people's cleanliness). A number of interesting findings emerged. Overall, we observed that generally, participants who more dispositional attributions (i.e., attributing their risk to factors such as age and own personal hygiene) rated their COVID risk to be significantly lower, whereas participants who made more situational attributions (i.e. attributing COVID-19 risk to government decision-making and other people's cleanliness) rated their risk significantly higher. Within this general trend, we then observed five typologies of risk attribution responses: intrinsic, behavioural, balanced, contextual and disengaged. Beyond the textual data, we also found that response typology significantly affected risk perception, in that those who exhibited a behavioural or intrinsic response, which were overwhelming attributed to dispositional factors, rated their likelihood of contracting coronavirus to be significantly lower than other response typologies. These findings may be useful in understanding the factors that affect (none) compliance with health advice to prevent the spread of COVID-19.

Whilst a body of rapidly emerging literature has assessed the psychological factors associated with the COVID-19 pandemic (e.g., Van Bavel et al., 2020), this study is the first to explore individual perception of ones' risk of contracting COVID-19, through a lens of risk attribution (Kelley, 1967, 1971) and locus of control (Wallston, 1992) theory. Whilst previous evidence has suggested that internally located agency of control is more likely to be associated with positive health behaviour change (Normam, Bennett, Smith \& Murphy, 1998; Seeman \& Seeman, 1983), our findings here extend this work in a timely, novel context. Importantly, our results show that the most common response typology in which participants made dispositional attributions (intrinsic), and thus demonstrate the highest level of internal local control, was not associated with highest adherence to relevant health behaviours in line with their perceived risk. However, the behavioural typology of response resides firmly 
within each individuals' locus of control and reinforces the ways in which individual action can protect against COVID-19.

On the opposite end of the spectrum, the disengaged and contextual response typologies position the risk, and therefore blame, of COVID-19 within more global and systemic forces. Therefore, this may mean that those who attribute their perceived risk of contracting COVID-19 to situational factors may not readily engage with preventative advice as actively as those who position the locus of control internally. Therefore our findings are mainly consistent with the wealth of previous literature that suggests that high internal locus of control promote positive engagement with health promotion (Norman, Bennett, Smith \& Murphy, 1998; Wallston, 1992). On the whole, these findings also echo Wallston and Wallston's (1981) assertion that internal control is associated with uptake of health behaviours and adherence to health advice.

Moreover, within the observed typology of responses, respondents who described positive uptake of health behaviours in response to the threat were generally positioned at either end of the perceived threat scale (i.e. they were categorised as generally either intrinsic \& disengaged). This supports previous work that assess responses to COVID-19, which demonstrated that the general population may find it difficult to fully engage with the severity of the global health risk (Van Bavel et al., 2020). Whilst the lowest behaviour response typologies are on different ends of the perceived threat scale, it is clear that the individuals in both these groups do not engage effectively with information about COVID-19 risk. For example, the intrinsic response group attribute COVID-19 risk to their own internal characteristics, and generally report a perception of little to no risk. Therefore, these respondents may not value the impact of health behaviours as a mechanism to reduce their risk of infection or infecting vulnerable others, as their attribution of risk is based on personal attributes rather than behavioural response. Furthermore, the most frequent response typology 
provided within our sample was categorised as disengaged. It may be argued that this group have not fully engaged with the health officials' information surrounding COVID-19, as this group believe that contracting the disease is inevitable, therefore the group generally do not endorse or adhere to beneficial information about health behaviours which would reduce the spread of the disease.

\section{Strengths of the present study}

This study demonstrated how perceptions of risk can be attributed to different sources, of which may be internal, external, or indeed both. The coexistence of both dispositional and situational attribution in one response aligns with Solomon's (1978) notion that dispositional and situational attributions are not simply inverses of one another. In this paper, we have applied a classic social psychological theory to a novel, timely global problem. Rapidly emerging literature continues to note the social psychological impact of COVID-19 in a plethora of contexts (e.g. Keyworth, 2020; Li, Yang, Dou, \& Cheung, 2020; Michie, 2020; Van Bavel et al., 2020). This paper contributes to the body of literature that assesses how individual perceptions of risk can be understood.

Moreover, in the weeks following the data collection of this study (March 2020), there were a number of useful resources aimed at people who satisfy the criteria of 'high risk' (e.g those with pre-existing health conditions, pregnant women, or people over the age of 70; (Unicef, 2020, Royal College of Nursing, 2020; NHS, 2020). However, relatively little is known about how 'typical risk' people make judgements about risk, which will inform behaviour. In this study, we tested a group of young, generally lower risk participants to investigate risk attribution of this sample. Therefore, these enquiries should now be extended in future work to more diverse, higher-risk groups, to test whether the typologies of response that we observed are replicated with a broader sample of participants. This will extend our understanding about how COVID-19 risk is individually judged. 


\section{Limitations of the present study}

Whilst this one cross-sectional study goes some way in exploring the ways in which people make attributions about their COVID-19 perceived risk, there are some methodological limitations that should be considered. For example, the majority of participants in our sample were young, female, and White. This means that our sample is inherently heterogeneous and relatively small. Therefore, the representativeness of these typologies may not be robust enough to be applied universally to all UK residents and beyond. Whilst preliminary research indicates that prevalence rates of COVID-19 are broadly equitable between men and women (Jin et al., 2020), there are reports, in the context of other health behaviours, to suggest that gender differences exist in risk perception (e.g., in cancer risk worry, McQueen et al., 2008, and smoking, Toll et al., 2008). Further, there are also gender differences in traits and constructs that impact risk perception, such as neuroticism (see Lynn \& Martin, 1977) which may impact COVID-19 fear (e.g., Lee et al., 2020).

Therefore, future work should interrogate gender differences further, particularly in the context of gender differences that may exist within health message compliance (e.g., Taraki \& Yano, 2010). The need for more diverse extensions and replications of this work is also important, given how other sociodemographic factors have been found to impact COVID-19 risk (Karmakar et al., 2021).

Whilst we have provided an account of risk responses typologies in response to COVID-19, there now remains scope for work to consider psychosocial interventions that can attempt to shift the locus of control. Our findings broadly suggest that an approach to risk that considers individual responsibility, capacity for behavioural prevention, and appreciation of the severity of the pandemic is the most promising in terms of positive behaviour change and 
compliance with advice. Work which encourages people to consider both the internal and external capacity of change, in response to the COVID-19, is now needed.

In summary, our findings reflect the range in responses to the perceived risk that COVD-19 presents. These response typologies follow a scale of perceived risk and dispositional versus situational attribution of risk. Our findings suggest the respondent groups who are least likely to engage with the positive behavioural changes required to fight the spread of COVID-19 and therefore can be used in efforts to increase compliance. It is also worth noting that data for this study were collected in March 2020, and in the past year the level of risk, behavioural adherence, and COVID-19 advice has varied substantially between countries. This likely means that the snapshot of COVID-19 risk perceptions presented here, in the earlier stages of the pandemic, may not directly replicate to the more progressed stages of the pandemic, particularly as some countries now begin to envisage a 'new normal' future beyond COVID-19. This study does, however, provide a useful insight into how people grapple with the issue of situating their own personal risk in the context of dispositional and situational attribution, which will be useful at moving forward, particularly as efforts turn towards 'health literacy' in COVID-19 and beyond (Paakkari \& Okan, 2020).

As Short and Mollborn (2015) note, however, a "social determinants" approach to health and behaviour change may be useful here in shifting focus away from individual attribution to more structural, overarching frameworks of health. In this paper, we take an individualised approach to risk attribution, assessing how one's own judgement about likelihood of contracting COVID-19 is formed. To extend these initial enquiries, future work may now consider a more systemic, global approach to the perception of risk, calling into question how larger groups attribute risk and adjust behaviour accordingly, in the context of this timely global pandemic. It should also be noted that data were collected in March 2020, pre-UK lockdown, at a time where concern for COVID-19 was beginning to grow. As the 
landscape of COVID risk and severity continues to rapidly change, in the UK and beyond, the contribution of individual risk attribution, health recommendation compliance, and the various social, cultural, and personal factors at play (van Bavel, 2020) should continue to be scrutinised. Finally, subsequent research may now wish to re-evaluate these response typologies as the severity of global responses to COVID-19 continues to progress

\section{References}

Axelrad, K. J. (1982). Locus of control and causal attributions as they relate to expectations for coping with a heart attack. California School of Professional Psychology. Los Angeles. Dissertation abstracts.

Bar-On, D. (1987). Causal Attributions and the Rehabilitation of Myocardial Infarction Victims. Journal of Social and Clinical Psychology: Vol. 5, Special Section: Interfaces, pp. 114-122. https://doi.org/10.1521/jscp.1987.5.1.114

Bruine de Bruin, W. (2021). Age differences in COVID-19 risk perceptions and mental health: Evidence from a national US survey conducted in March 2020. The Journals of Gerontology: Series B, 76(2), e24-e29.

Brown, E., Gray, R., Monaco, S. L., O'Donoghue, B., Nelson, B., Thompson, A., ... \& McGorry, P. (2020). The potential impact of COVID-19 on psychosis: a rapid review of contemporary epidemic and pandemic research. Schizophrenia research.

Dunning, A., Travis, E., \& Pownall, M. (2021, April 21). Dispositional and situational attribution of COVID-19 risk: A content analysis of response typology. Retrieved from https://osf.io/4ckn3/ Fereday, J., \& Muir-Cochrane, E. (2006). Demonstrating rigor using thematic analysis: A hybrid approach of inductive and deductive coding and theme development. International Journal of Qualitative Methods, 5(1), 80-92. 
Ferguson, N. \&... Ghani., C. (2020) Impact of non-pharmaceutical interventions (NPIs) to reduce COVID- 19 mortality and healthcare demand. https://doi.org/10.25561/77482

Gerhold, L. (2020, March 25). COVID-19: Risk perception and Coping strategies. https://doi.org/10.31234/osf.io/xmpk4

Heider, F. (1958). The psychology of interpersonal relations. Psychology Press.

Hirsch, J. K., Wolford, K., LaLonde, S. M., Brunk, L., \& Parker-Morris, A. (2009). Optimistic explanatory style as a moderator of the association between negative life events and suicide ideation. Crisis: The Journal of Crisis Intervention and Suicide Prevention, 30(1), 48.

Janoff-Bulman, R. (1979). Characterological versus behavioral self-blame: Inquiries into depression and rape. Journal of personality and social psychology, 37(10), 1798.

Jin, J. M., Bai, P., He, W., Wu, F., Liu, X. F., Han, D. M., ... \& Yang, J. K. (2020). Gender differences in patients with COVID-19: Focus on severity and mortality. Frontiers in Public Health, 8, 152.

Karmakar, M., Lantz, P. M., \& Tipirneni, R. (2021). Association of social and demographic factors with COVID-19 incidence and death rates in the US. JAMA network open, 4(1), e2036462-e2036462.

Kelley, H. H. (1967). Attribution theory in social psychology. In D. Levine (Ed.), Nebraska Symposium on Motivation (Vol 15, pp. 192-238). Lincoln: University of Nebraska Press.

Kelley, H. H. (1971). Attribution in social interaction. Morristown, NJ: General Learning Press.

Keyworth, C. (2020). Keep calm, and listen to the experts. The Psychologist. Retrieved from: https://thepsychologist.bps.org.uk/keep-calm-and-listen-experts 
Kuckartz, U. (2019). Qualitative text analysis: A systematic approach. In Compendium for Early Career Researchers in Mathematics Education (pp. 181-197). Springer, Cham.

Kuper-Smith, B. J., Doppelhofer, L. M., Oganian, Y., Rosenblau, G., \& Korn, C. (2020, March 19). Optimistic beliefs about the personal impact of COVID-19. https://doi.org/10.31234/osf.io/epcyb

Lee, S. A., \& Crunk, E. A. (2020). Fear and psychopathology during the COVID-19 crisis: neuroticism, hypochondriasis, reassurance-seeking, and coronaphobia as fear factors. OMEGA-Journal of Death and Dying, 0030222820949350.

Li, J. B., Yang, A., Dou, K., \& Cheung, R. Y. (2020). Self-control moderates the association between perceived severity of the coronavirus disease 2019 (COVID-19) and mental health problems among the Chinese public.

Lynn, R., \& Martin, T. (1997). Gender differences in extraversion, neuroticism, and psychoticism in 37 nations. The Journal of Social Psychology, 137(3), 369-373.

Maddux, J. E., Brawley, L., \& Boykin, A. (1995). Self efficacy and healthy behavior: Prevention, promotion, and detection. In J. E. Maddux (Ed.), Self-efficacy, adaptation, and adjustment: Theory, research, and application. The Plenum series in social/clinical psychology (pp. 173-202). New York: Plenum Press. Miller, D. T., \& Ros

Marteau, T. M. (1995). Health beliefs and attributions. In Health psychology (pp. 3-20). Springer, Boston, MA.

McBride, O., Murphy, J., Shevlin, M., Gibson-Miller, J., Hartman, T. K., Hyland, P., ... \& Stocks, T. V. (2020). Monitoring the psychological impact of the COVID-19 pandemic in the general population: an overview of the context, design and conduct of the COVID-19 Psychological Research Consortium (C19PRC) Study. 
McGorry, P. (2020). The potential impact of COVID-19 on psychosis: A rapid review of contemporary epidemic and pandemic research. Schizophrenia Research.

Michie, S. (2020) Behavioural science must be at the heart of the public health response to covid-19 The BMJ Opinion. https://blogs.bmj.com/bmj/2020/02/28/behavioural-

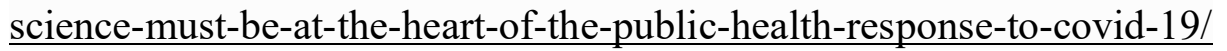

Murphy, K., Williamson, H., Sargeant, E., \& McCarthy, M. (2020). Why people comply with COVID-19 social distancing restrictions: Self-interest or duty?. Australian \& New Zealand Journal of Criminology, 0004865820954484.

NHS (2020) Coronavirus: Advice for everyone. NHS.uk Retrieved from https://www.nhs.uk/conditions/coronavirus-covid-19/

NHS (2020). Advice for people at high risk Coronavirus (COVID-19). NHS.uk Retrieved from: https://www.nhs.uk/conditions/coronavirus-covid-19/advice-for-people-at-high$\underline{\text { risk/ }}$

Norman, P., Bennett, P., Smith, C., \& Murphy, S. (1998). Health locus of control and health behaviour. Journal of health psychology, 3(2), 171-180.

O'Connor, D. B., Aggleton, J. P., Chakrabarti, B., Cooper, C. L., Creswell, C., Dunsmuir, S., ... \& Jones, M. V. (2020). Research priorities for the COVID-19 pandemic and beyond: A call to action for psychological science. British Journal of Psychology. 111(4).

O'hea, E. L., Grothe, K. B., Bodenlos, J. S., Boudreaux, E. D., White, M. A., \& Brantley, P. J. (2005). Predicting medical regimen adherence: The interactions of health locus of control beliefs. Journal of Health Psychology, 10(5), 705-717.

Oosterhoff, B., \& Palmer, C. (2020, March 23). Psychological Correlates of News Monitoring, Social Distancing, Disinfecting, and Hoarding Behaviors among US Adolescents during the COVID-19 Pandemic. https://doi.org/10.31234/osf.io/rpcy4 
Paakkari, L., \& Okan, O. (2020). COVID-19: health literacy is an underestimated problem. The Lancet Public Health, 5(5), e249-e250.

Peterson, C., Seligman, M., \& Vaillant, G. (1988). Pessimistic explanatory style is a risk factor for physical illness: A 35-yearlongitudinal study. Journal of Personality and Social Psychol-ogy, 55, 23-27.

Peterson, C., \& Villanova, P. (1988). An expanded attributional style questionnaire. Journal of Abnormal Psychology, 97(1), 87-89.

Rajkumar, R. P. (2020). COVID-19 and mental health: A review of the existing literature. Asian journal of psychiatry, 102066.

Roozenbeek, Jon, Claudia R. Schneider, Sarah Dryhurst, John Kerr, Alexandra LJ Freeman, Gabriel Recchia, Anne Marthe van der Bles, and Sander van der Linden. "Susceptibility to misinformation about COVID-19 around the world." Royal Society Open Science 7, no. 10 (2020): 201199

Rotter, J. B. (1954). Social learning and clinical psychology.

Rowe, J. L., Montgomery, G. H., Duberstein, P. R., \& Bovbjerg, D. H. (2005). Health locus of control and perceived risk for breast cancer in healthy women. Behavioral Medicine, 31(1), 33-42.

Royal College of Nursing (2020) COVID-19 (coronavirus) FAQs. Retrieved from https://www.rcn.org.uk/get-help/rcn-advice/covid-19

Seeman, M., \& Seeman, T. E. (1983). Health behavior and personal autonomy: A longitudinal study of the sense of control in illness. Journal of health and social behavior, 144-160.

Short, S. E., \& Mollborn, S. (2015). Social determinants and health behaviors: conceptual frames and empirical advances. Current opinion in psychology, 5, 78-84. 
Sobol, M., Blachnio, A., \& Przepiórka, A. (2020). Time of pandemic: Temporal perspectives related to compliance with public health regulations concerning the COVID-19 pandemic. Social Science \& Medicine, 265, 113408.

Solomon, S. (1978). Measuring dispositional and situational attributions. Personality and social psychology bulletin, 4(4), 589-594.

Stanley, M., Seli, P., Barr, N., \& Peters, K. (2020, March 25). Analytic-Thinking Predicts Hoax Beliefs and Helping Behaviors in Response to the COVID-19 Pandemic. https://doi.org/10.31234/osf.io/7456n

Takaki, J., \& Yano, E. (2006). Possible gender differences in the relationships of self-efficacy and the internal locus of control with compliance in hemodialysis patients. Behavioral Medicine, 32(1), 5-11.

Tenforde, M. W., Fisher, K. A., \& Patel, M. M. (2021). Identifying COVID-19 risk through observational studies to inform control measures. JAMA. http://doi.org/10.1001/jama.2021.1995

Toll, B. A., Salovey, P., O'Malley, S. S., Mazure, C. M., Latimer, A., \& McKee, S. A. (2008). Message framing for smoking cessation: The interaction of risk perceptions and gender. Nicotine \& tobacco research, 10(1), 195-200.

Unicef (2020) Everything you need to know about washing your hands to protect against coronavirus (COVID-19) Retrieved from: https://www.unicef.org/coronavirus/everything-you-need-know-about-washing-yourhands-protect-against-coronavirus-covid-19

Valins, S., \& Nisbett, R. E. (1971). Attribution process in the development and treatment of emotional disorders. Morristown, NJ: General Learning Press 
Van Bavel, J. J., Baicker, K., Boggio, P. S., Capraro, V., Cichocka, A., Cikara, M., ... \& Drury, J. (2020). Using social and behavioural science to support COVID-19 pandemic response. Nature Human Behaviour, 1-12.

Wallston, K. A. (1992). Hocus-pocus, the focus isn't strictly on locus: Rotter's social learning theory modified for health. Cognitive therapy and research, 16(2), 183-199.

Wallston, K. A. (1992). Hocus-pocus, the focus isn't strictly on the locus: Rotter social learning theory modified for health. Cognitive Therapy and Research, 16, 183-199.

Weiner, B. (1985). An attributional theory of achievement motivation and emotion. Psychological review, 92(4), 548.

Weiner, B., Nierenberg, R., \& Goldstein, M. (1976). Social learning (locus of control) versus attributional (causal stability) interpretations of expectancy of success 1. Journal of Personality, 44(1), 52-68.

Wong, C. M. L., \& Jensen, O. (2020). The paradox of trust: perceived risk and public compliance during the COVID-19 pandemic in Singapore. Journal of Risk Research, $1-10$

Xu, J., \& Peng, Z. (2015). People at risk of influenza pandemics: The evolution of perception and behavior. PloS one, 10(12), e0144868. 


\section{Figures and Tables}

Table 1. Comparisons between risk perception of all response typologies

\begin{tabular}{llccccc}
\hline & & Mean Difference & $\mathrm{SE}$ & $\mathrm{t}$ & \multicolumn{2}{c}{$\mathrm{p}$ bonf } \\
\hline Behavioural & Intrinsic & -5.42 & 8.12 & -0.67 & 1.00 & \\
& Balanced & -33.41 & 7.15 & -4.67 & $<.001$ & $* * *$ \\
& Contextual & -46.08 & 7.52 & -6.12 & $<.001$ & $* * *$ \\
& Disengaged & -51.36 & 7.18 & -7.15 & $<.001$ & $* * *$ \\
Intrinsic & Balanced & -27.99 & 6.83 & -4.10 & $<.001$ & $* * *$ \\
& Contextual & -40.66 & 7.23 & -5.63 & $<.001$ & $* * *$ \\
& Disengaged & -45.94 & 6.87 & -6.69 & $<.001$ & $* * *$ \\
Balanced & Contextual & -12.67 & 6.11 & -2.07 & 0.40 & \\
& Disengaged & -17.95 & 5.68 & -3.16 & 0.02 & $*$ \\
& Disengaged & -5.28 & 6.15 & -0.86 & 1.00 & \\
\hline
\end{tabular}

Note. P-value adjusted for comparing a family of 5

$* \mathrm{p}<.05, * * \mathrm{p}<.01, * * * \mathrm{p}<.001$

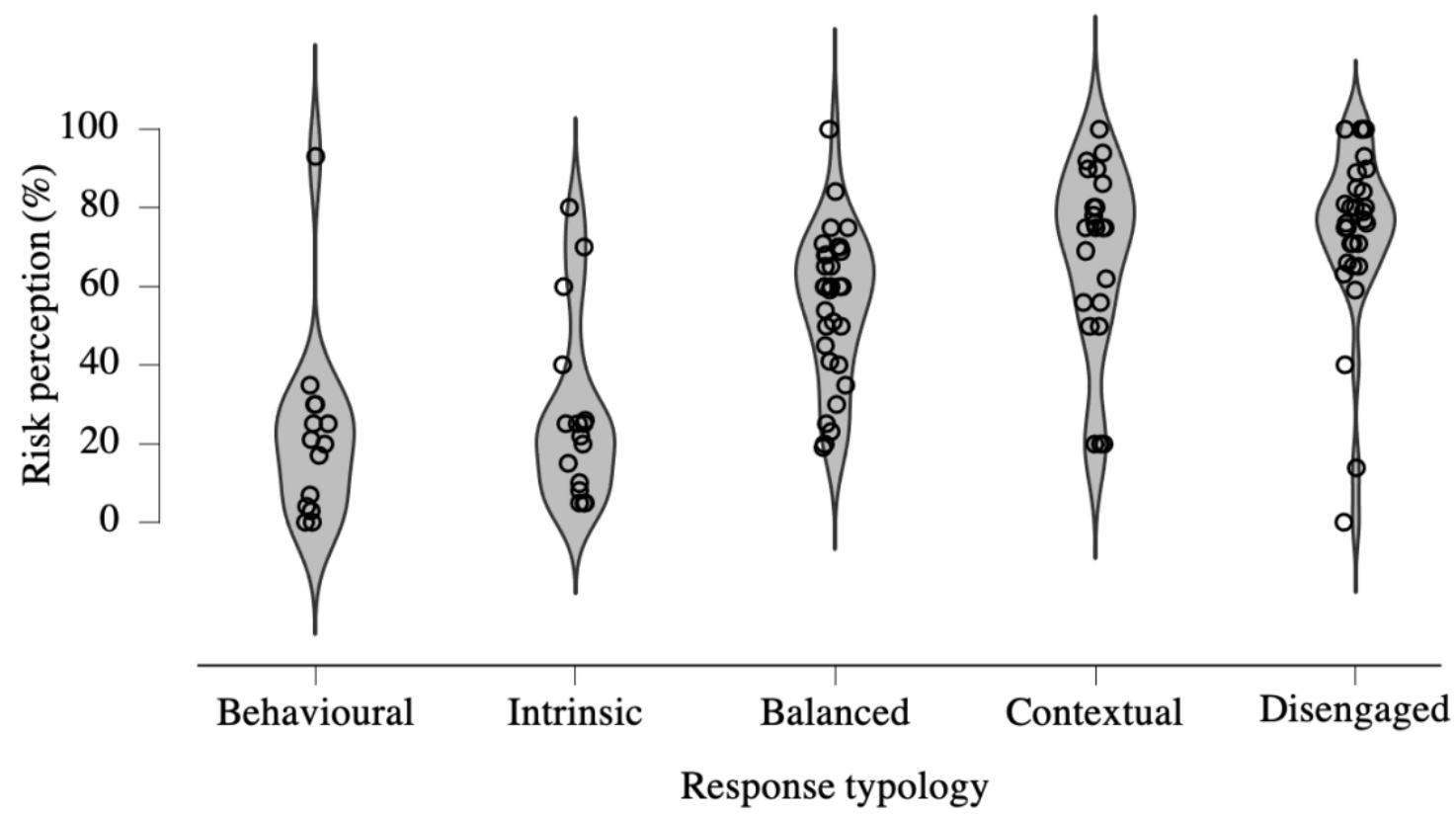

Figure 1. Mean perceived COVID risk (\%) by response typology. Error bars show $95 \%$ confidence intervals. 